Itinéraires Itinéraires

Littérature, textes, cultures

\title{
Michael Bristol, Kathleen McLuskie et Christopher Holmes, Shakespeare and Modern Theatre, The Performance of Modernity
}

Londres et New York, Routledge, 2001.

\section{Estelle Rivier}

\section{(2) OpenEdition}

\section{Journals}

Édition électronique

URL : http://journals.openedition.org/itineraires/1769

DOI : 10.4000/itineraires. 1769

ISSN : 2427-920X

\section{Éditeur}

Pléiade

Édition imprimée

Date de publication : 1 décembre 2010

Pagination : 188-191

ISBN : 978-2-296-13183-5

ISSN : 2100-1340

\section{Référence électronique}

Estelle Rivier, « Michael Bristol, Kathleen McLuskie et Christopher Holmes, Shakespeare and Modern Theatre, The Performance of Modernity », Itinéraires [En ligne], 2010-4 | 2010, mis en ligne le 01 décembre 2010, consulté le 22 septembre 2020. URL : http://journals.openedition.org/itineraires/1769 ; DOI : https://doi.org/10.4000/itineraires. 1769

Ce document a été généré automatiquement le 22 septembre 2020.

\section{c)}

Itinéraires est mis à disposition selon les termes de la licence Creative Commons Attribution - Pas d'Utilisation Commerciale - Pas de Modification 4.0 International. 


\section{Michael Bristol, Kathleen McLuskie et Christopher Holmes, Shakespeare and Modern Theatre, The Performance of Modernity}

Londres et New York, Routledge, 2001.

\section{Estelle Rivier}

\section{RÉFÉRENCE}

Michael Bristol, Kathleen McLuskie et Christopher Holmes, Shakespeare and Modern Theatre, The Performance of Modernity, Londres et New York, Routledge, 2001.

1 À une époque où les œuvres de Shakespeare se présentent sous les formes les plus diverses, en particulier, au cinéma et sur les scènes de théâtre, la critique soulève certaines questions: ces modes de transmission de la littérature renaissante supplanteraient-elles les versions manuscrites? La connaissance du public d'aujourd'hui serait-elle mieux alimentée par les interprétations et relectures scénographiées de ces œuvres? L'ouvrage, Shakespeare and Modern Theatre, The Performance of Modernity, regroupe des essais d'auteurs d'horizons variés qui explorent les approches ayant modernisé le texte original de Shakespeare, que ce soit par les mises en scène, les traductions, l'histoire ou les courants esthétiques et idéologiques.

Dans une introduction intitulée "Shakespeare and Modernism», Hugh Grady, situe tout d'abord chronologiquement les termes ou courants appelés "postmoderne » et " moderne ». Si l'on considère que la modernité de Shakespeare s'est renforcée à partir des années 1950 parallèlement à un renouveau artistique littéraire général, ce type de modernité, que Grady qualifie de "high modernism", ne peut être communément nommé «moderne». Bien d'autres termes tels que «modernisation» ou «modernisme » et surtout « postmodernisme » établissent les critères de style propres 
à un moment donné de l'histoire d'un texte. À travers ces courants, notre perception de l'œuvre de Shakespeare a considérablement évolué : les textes ayant été mis en espace, lus en termes de symboles, de mythes et d'images, avant d'être considérés comme des récits. Ce phénomène s'est répercuté sur les mises en scène théâtrales et cinématographiques. Grady réfute en cela l'assertion de T.S. Eliot selon laquelle ce serait le caractère "primitif » de Shakespeare, c'est-à-dire sa proximité avec les rites anciens prémodernes, qui aurait rendu son œuvre moderne. Il appuie son argumentation sur deux productions «modernes », celle de Hamlet, par Edward Gordon Craig en 1912 et celle de Macbeth par Orson Welles en 1936. Grady se hasarde finalement à proposer une définition d'un Shakespeare postmoderne. Celui-ci serait formé à l'intérieur du Shakespeare moderne tardif comme s'il s'agissait d'un effet de style artistique. Le courant postmoderne ne serait en quelque sorte qu'une extension du modernisme.

3 C'est en relevant certains exemples de lectures du "sous-texte" des pièces de Shakespeare que Paul Yachnin développe son propos (par exemple, Goodnight Desdemona, Good Morning Juliet d'Anne-Marie MacDonald). Paul Yachnin nomme cette tendance la politique du "bardicide", évoquant en incipit l'approche critique de $L a$ Mégère Apprivoisée par Charles Marowitz. Cette comédie dépréciée par la gent féminine fut l'objet d'une réécriture où l'on assistait, en son dénouement, au viol de Katherine par Baptista et par son beau-père. Il fonde ensuite son discours sur les approches critiques de Pierre Bourdieu et de Mikhail Bakhtin. L'attrait que suscitent les pièces de Shakespeare est à l'origine du renouvellement constant des mises en scène qui, non seulement revisitent l'idéologie élisabéthaine, mais en proposent aussi une glose contemporaine.

4 Catherine Graham s'interroge, quant à elle, sur le statut professionnel de l'acteur dit «moderne ». Dans son essai, elle compare les interprétations de Hamlet par olivier et Gielgud dont les partis pris se situent aux antipodes l'un de l'autre. Hamlet est, semblet-il, le rôle de Shakespeare permettant de légitimer les compétences de l'acteur moderne car le tempérament d'Hamlet et le dilemme auquel il est confronté reflètent l'expérience de la subjectivité. L'évolution de l'interprétation de ce rôle va de pair avec la perception nouvelle que l'on a de la modernité de Shakespeare. Se libérant de certains modes d'interprétation prémodernes, les acteurs ont affirmé leur statut professionnel. À l'image de Hamlet, l'acteur dépasse les limites du rôle traditionnel pour inventer une fiction qui lui permette d'explorer une autre façon d'être dans le monde. Ses préoccupations demeurent celles d'être le plus plausible possible dans son interprétation du rôle. Il vise aussi à une excellence et à un inédit, qui se nourrissent, tous deux, du passé. Dans les dernières pages de son article, Catherine Graham détaille la façon dont les deux Hamlet, le premier par Olivier et le second par Gielgud, sont une leçon de théâtre professionnel: que ce soit dans la dichotomie corps/esprit ou dans le rapport enfant/parent, les approches d'olivier et de Gielgud sont divergentes, même si pour chaque interprète Hamlet demeure la référence de l'acteur moderne en devenir.

5 En tant qu'universitaire français, Jean-Michel Déprats puise ses références dans le théâtre contemporain de son pays et illustre son propos d'exemples issus de la scène française. La thèse majeure de son analyse est de rappeler le caractère «in-fini » de la traduction et, par là même, sa fugacité. Le théâtre, par essence est un arc tendu entre le passé et le présent, au-delà de tout temps donc. Déprats explore ensuite quelques techniques de traduction : l'une, par exemple, mettrait l'accent sur l'époque de l'auteur 
ou sur celle du lectorat/public. Une autre, plus historique, consisterait à mettre en valeur ce qui est fini, unique et discontinu. Jean-Michel Déprats souligne qu'il n'existe pas de traduction française contemporaine de l'époque de Shakespeare. Il y a donc eu de nombreuses adaptations depuis le XVIII ${ }^{e}$ siècle et de fait, l'on glisse de plus en plus vers des versions en adéquation avec l'époque où elles sont jouées. Finalement, ce constat est plutôt positif puisque traduire ouvre de nouvelles perspectives d'interprétation du texte original qui revêt alors d'autres couleurs au gré des relectures/réécritures. L'auteur montre l'adaptabilité du texte shakespearien au temps où il "renaît». De fait, fixer une traduction pour toujours relèverait de l'utopie éditoriale. Une traduction est une liaison entre les âges, non un simple moyen de transport. L'objet « texte » est fluctuant : il nous échappe dès lors qu'on croirait l'avoir conquis, ce qui en fait sa modernité.

6 Sarah Werner retrace le parcours de la branche féminine de la RSC («Women's Group ») née dans les années 1980 à la suite d'un mouvement protestataire mené par les actrices de la RSC : Fiona Shaw, Genista McIntosh, Lindsay Duncan, Susan Todd, Deborah Levy et Juliet Stevenson. Cette période brève de deux années (1986-1987) fut considérée comme une intersection entre le féminisme et le théâtre shakespearien moderne. Elle s'acheva avec la mise en scène au Barbican Pit de Heresies, une pièce de Deborah Levy. La principale actrice à l'origine de ce mouvement fut Susan Todd, mais ses efforts furent voués à l'échec notamment parce que la RSC revendiquait une politique de neutralité entre les sexes. Enfin, dans quelle mesure les femmes dont l'emploi et, par conséquent, les ressources financières dépendaient de la gestion masculine de la RSC pouvaient-elles s'attendre à être soutenues par leur direction? Ainsi, la modernité de Shakespeare repose aussi sur le rejet d'un excès de politique ouvertement féministe.

7 Les metteurs en scène contemporains sont préoccupés par la valeur performative des pièces de Shakespeare, autrement dit la faisabilité de leur mise en espace en adéquation avec un sens toujours valable. W. B. Worthen appuie son étude sur l'exemple du Nouveau Théâtre du Globe à Londres, lieu qui permet de saisir la performance théâtrale telle l'incarnation du passé mise en texte. Le Nouveau Globe est une façon de trouver le sens original du texte par sa mise en œuvre dans les conditions de l'époque de sa création, à la Renaissance anglaise. On pourrait alors gloser le terme " performativity " qu'utilise Worthen par «force du texte en scène ». Sont alors remises en question les vertus de la représentation théâtrale dont le caractère éphémère est lié à son époque, à un moment donné de l'histoire. En témoignent les mises en scène de Garrick : louées à leur époque, elles sont critiquées de nos jours. En outre, modifier notre lecture d'un texte ouvre des possibilités d'interprétation scénique (féministes, épiques, par exemple). Inversement, les mises en scène révèlent un sémantisme textuel nouveau. Enfin, la modernisation de la mise en espace d'un texte crée inévitablement des incongruités scéniques: certaines didascalies ne peuvent être respectées. Ainsi, si la reconstitution historique des conditions de jeu renaissant peut aider à la compréhension d'un texte, elle n'empêche pas les tentatives de modernisation scénographique des œuvres d'être justifiées, ne serait-ce que pour réaffirmer leur " performativité ».

8 À l'image d'Edward Bond qui réécrivit Le Roi Lear afin de l'adapter à son temps, le metteur en scène ukrainien, Les' Kurbas, a mis en scène quatre variations de Macbeth en 1924. Dans son étude, Irena R. Makaryk commente la quatrième version de cette suite. 
Les' Kurbas était en quête d'une nouvelle grammaire théâtrale pour la société soviétique ukrainienne. Il présenta une première version de Macbeth en ukrainien, en 1919-1920. En 1924, alors que la stabilité politique était a priori revenue, avec la création d'une république soviétique ukrainienne, les débats concernant les liens à tisser entre politique, arts et nation fleurissaient dans les journaux. Les courants symboliste, futuriste, néoclassique, la VAPLITE (Académie libre de littérature prolétaire) et bien d'autres esthétiques étaient au cœur des discours. Pour Les' Kurbas, il fallait un style avant-gardiste afin que le thêâtre soit moderne, nouveau et pertinent. Son Macbeth obtint un vif succès. Théâtre illusionniste, fait de contrastes, de juxtapositions de styles, de montages, d'effets minimalistes, cette interprétation portait un regard ironique sur la fable morale du héros éponyme ambitieux. Ce type de pratique métadramatique engagea un certain nombre d'interrogations : quelle vision satirique était visée par la mise en scène? Comment étaient traitées les questions sur le pouvoir, la conscience, la loyauté, la complicité silencieuse, ou la destruction de l'innocence avec, en arrière-plan de la scène, la mort récente de Lénine ? Quelle était la morale de cette mise en scène ou le pouvoir du tsar (Duncan) n'était pas meilleur que celui du régime soviétique incarné par les Malcom et Macduff? Kurbas souhaitait re-concevoir la notion de théâtre, même s'il lui fut reproché de n'en faire qu'une forme artistique trop abstraite, un art pour l'art, un système de signes détachés de la réalité.

L'œuvre de Shakespeare est aujourd'hui produite pour un marché culturel des plus variés. Si on en traduit les scripts et si on la retravaille pour qu'elle véhicule un propos contemporain, pourquoi garder la fable et ne pas tenter une tout autre écriture fondée sur une intrigue nouvelle ? C'est peut-être que la force ou les valeurs performatives du texte sont irremplaçables. L'enchevêtrement des actions confère un rythme aux pièces ; de même, la veine historique qui les parcourt renforce les partis pris prônés dans les mises en scène contemporaines où le cycle infernal de l'histoire est souvent mis en exergue.

Cet ouvrage dense et complexe nous met au défi de comprendre les enjeux de la modernité de Shakespeare. De nombreuses cultures s'approprient cette littérature, ce qui au contraire de la trahir, ne fait que la rendre plus universelle, à l'instar des propres visées de leur auteur renaissant qui déjà mélangeaient styles, pays, langages, cultures et idéologies après s'être lui aussi nourri du travail de ses prédécesseurs.

\section{AUTEURS \\ ESTELLE RIVIER}

Université du Maine 